\title{
Recurrent hypoglycemia: boosting the brain's metabolic flexibility
}

\author{
Marina Litvin, ${ }^{1}$ Amy L. Clark, ${ }^{2}$ and Simon J. Fisher ${ }^{1,3}$
}

${ }^{1}$ Division of Endocrinology, Metabolism, and Lipid Research, Department of Medicine, ${ }^{2}$ Division of Endocrinology and Diabetes, Department of Pediatrics, and ${ }^{3}$ Department of Cell Biology and Physiology, Washington University, St. Louis, Missouri, USA.

\begin{abstract}
For people with diabetes, recurrent episodes of hypoglycemia limit the brain's ability to sense dangerously low blood sugar levels. In this issue of the JCI, the mechanisms behind this clinical problem of hypoglycemia unawareness are addressed by Herzog et al. The authors provide compelling evidence that recurrent hypoglycemia enhances transport of lactate into the brain and, although not itself a major alternative fuel source, lactate may preserve neuronal function during hypoglycemia by maintaining neuronal glucose metabolism. These findings redefine our understanding of the brain's metabolic adaptations that result from recurrent hypoglycemia.
\end{abstract}

The clinical problem of hypoglycemia

Despite decades of research, diabetes remains a life-threatening disease with an increasing prevalence worldwide. Clinical studies have consistently shown that glucose-lowering interventions decrease the rate of diabetic complications, including nephropathy, retinopathy, and neuropathy $(1,2)$. Unfortunately, improved glycemic control invariably increases the incidence of hypoglycemia $(1,2)$. Hypoglycemia is therefore the limiting factor for blood glucose management in patients with diabetes and is a major barrier to the attainment of microvascular benefits associated with tight glycemic control. Hypoglycemia is very common, on average occurring more than twice a day in patients with type 1 diabetes (3). When severe, hypoglycemia can cause brain damage $(4,5)$, long-term cognitive dysfunction (6), and even sudden death (7). Hypoglycemia-related fatalities account for death in up to $10 \%$ of patients with type 1 diabetes (8).

Recurrent episodes of hypoglycemia lead to both a decreased sympathoadrenal response to hypoglycemia and an impaired ability to recognize it (i.e., hypoglycemia unawareness), together termed hypoglycemia-associated autonomic failure (HAAF) (9). The presence of HAAF in patients with diabetes substantially increases the risk for severe hypoglycemia (9). The mechanisms by which recurrent hypoglycemia

Conflict of interest: Simon J. Fisher is a member of Merck's speakers' bureau.

Citation for this article: J Clin Invest. 2013; 123(5):1922-1924. doi:10.1172/JCI69796. causes HAAF are not fully understood and remain an active area of research interest. As reviewed elsewhere $(9,10)$, multiple mechanisms have been proposed to explain the impaired brain glucose sensing associated with recurrent episodes of hypoglycemia, including the actions of hormones released during these episodes (cortisol, epinephrine, opioids), enhanced cerebral glucose metabolism (transport/ phosphorylation/oxidation), or increased cerebral storage of glycogen, increased GABAergic tone, and the use of alternative fuels (e.g., $\beta$-hydroxybutyrate, acetate, lactate) to support brain metabolism. These proposed mechanisms are not mutually exclusive; indeed, given the body's numerous responses to hypoglycemia, multiple contributing factors likely mediate brain adaptations characteristic of HAAF.

\section{Hypoglycemia unawareness and the alternative fuel hypothesis}

The alternative fuel hypothesis, although first suggested more than 20 years ago, has recently become in vogue as a plausible mechanism that contributes to hypoglycemia unawareness. According to this paradigm, recurrent hypoglycemia induces an adaptive response in which the blood-brain barrier, neurons, and/or astrocytes increase their capacity to uptake fuels other than glucose, which contributes to hypoglycemia unawareness by sustaining neuronal metabolism during hypoglycemia. Supporting this notion, an upregulation of monocarboxylic acid (acetate) transport has been shown to maintain brain energetics during hypoglycemia in patients with type 1 diabetes (11). Also, patients with diabetes and hypoglycemia unawareness have been shown to have increased brain lactate levels (12). Consistent with this alternative fuel hypothesis is the finding that lactate, normally released from adjacent astrocytes, may be preferred over glucose as a neuronal energy substrate (13).

Some caveats to this alternative substrate hypothesis are noteworthy. Most studies demonstrating the CNS benefits of using lactate as an alternative fuel during hypoglycemia involve lactate infusions that reach supraphysiological lactate levels. Since lactate levels change little during hypoglycemia, it remains unclear whether physiological levels can provide sufficient metabolic substrate to maintain cerebral metabolism (even in the recurrently hypoglycemic-adapted brain). Additionally, although increased brain lactate in the setting of hypoglycemia unawareness has been demonstrated (12), other studies have indicated that increased lactate uptake alone is not of sufficient magnitude to offset the profound energy deficit caused by hypoglycemia (14).

\section{Lactate preserves neuronal metabolism following recurrent hypoglycemia}

In this issue of the JCI, Herzog et al. (15) explore the adaptations in brain metabolism that occur in rats exposed to 3 days of antecedent recurrent hypoglycemia $(3 \mathrm{dRH})$, a well-established rodent model of hypoglycemia unawareness and impaired counterregulation. By using labeled lactate infusions and NMR to trace lactate's metabolic fate, the authors demonstrate that $3 \mathrm{dRH}$-exposed rats were able to significantly increase neuronal uptake of lactate during hypoglycemia compared with controls. Thus, consistent with the alternative fuel hypothesis, brain adaptation to recurrent hypoglycemia increases the brain's capacity (i.e., metabolic flexibility) for alternative substrate uptake during hypoglycemia. An unexpected finding was that increased lactate influx was matched 
A

\section{B}

Acute hypoglycemia + lactate

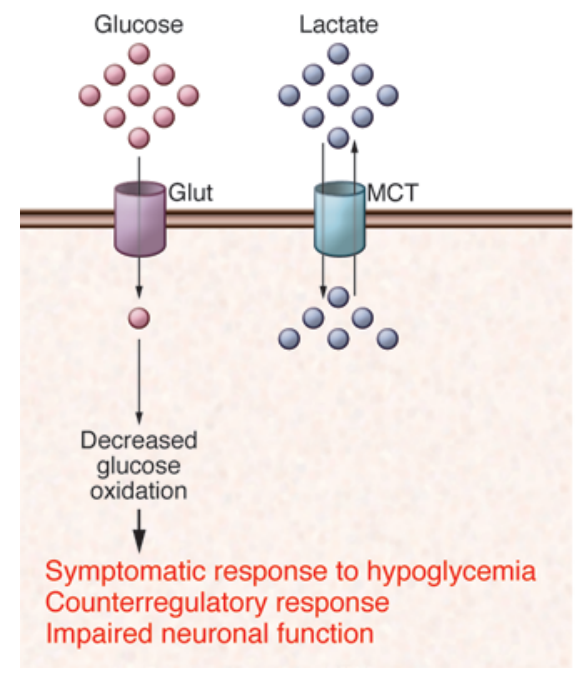

Antecedent recurrent hypoglycemia preconditioning Acute hypoglycemia + lactate

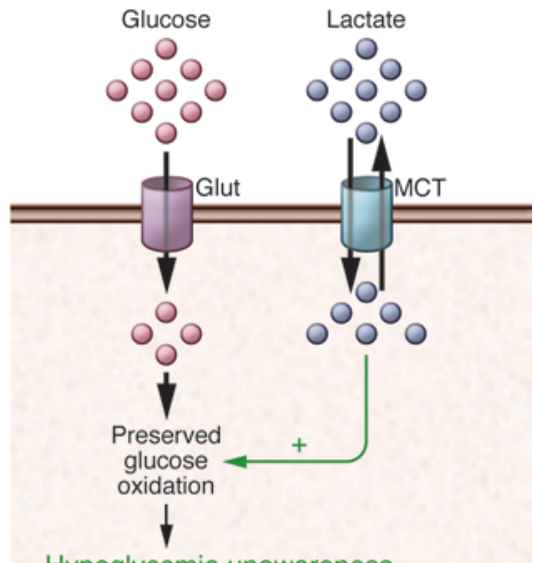

Hypoglycemia unawareness Impaired counterregulatory response Preserved neuronal function

\section{Figure 1}

Recurrent hypoglycemia preconditioning increases the brain's capacity for glucose and lactate uptake during hypoglycemia. Herzog et al. (15) traced the metabolic fate of infused lactate during insulin-induced hypoglycemia in the brains of rats. The figure depicts neuronal cells during conditions of acute hypoglycemia and lactate infusion either $(\mathbf{A})$ without or $(\mathbf{B})$ with antecedent hypoglycemic preconditioning. During acute hypoglycemia, intracellular glucose levels decline markedly and limit glucose oxidation and ATP generation, even in the presence of lactate infusion. With very low intracellular glucose levels, there is a relatively large transmembrane glucose gradient, consistent with the notion that glucose transport is the rate-limiting step for brain glucose metabolism during hypoglycemia. The normal responses to hypoglycemia include symptoms of hypoglycemia (e.g., hypoglycemia awareness) associated with a brisk counterregulatory response. If hypoglycemia is severe, however, neuronal function can be impaired. The recurrent hypoglycemia-preconditioned brain, on the other hand, demonstrated an increased capacity for glucose transport (as depicted by bold arrow), which prevented such a marked decline in intracellular glucose. Preconditioning thus maintains neuronal glucose oxidation and preserves brain function during hypoglycemia but consequently leads to the clinical problem of hypoglycemia unawareness and impaired sympathoadrenal responses. Preconditioning also increased the capacity for lactate transport via monocarboxylic acid transporters (MCT) as depicted by bold arrows. Although, with only a small lactate gradient in the brain, increased lactate influx was matched by lactate efflux, and lactate was not a significant alternative fuel substrate. The authors' propose that the infused lactate may have acted as a metabolic regulator that helped to preserve glucose metabolism during hypoglycemia. A better understanding of the brain's adaptations to recurrent hypoglycemia may help prevent the clinical conditions of hypoglycemia unawareness and impaired stress responses and that occur in patients with diabetes. Glut, glucose transporter.

by increased lactate efflux and not associated with increased brain lactate oxidation. By their calculations, the enhanced lactate transport was insufficient to support CNS metabolism. So if it was not metabolized, what was the role of lactate in the brains of the $3 \mathrm{dRH}$-treated rats? The elevated lactate transport in the $3 \mathrm{dRH}$ animals was associated with a sustained rate of neuronal glucose oxidation during hypoglycemia, that is, it prevented the normal fall in brain glu- cose oxidation that occurs during hypoglycemia (15). Thus, Herzog et al. have added a novel twist to the recurrent hypoglycemia alternative fuel hypothesis: in the recurrently hypoglycemic-adapted brain, lactate is not an alternative fuel substrate but perhaps serves as a "metabolic regulator" that preserves glucose metabolism during hypoglycemia (Figure 1).

In addition to enhancing glucose metabolism during hypoglycemia following
$3 \mathrm{dRH}$, the authors demonstrate that lactate also has a functional role in preserving cortical responses to forepaw stimulation. $3 \mathrm{dRH}$-exposed rats had a reduced cortical forepaw response during hypoglycemia, which normalized when they received lactate (15). These findings complement the authors' metabolic data, indicating that recurrent hypoglycemia leads to adaptations of both glucose and lactate uptake that preserve normal neuronal function during hypoglycemia.

Exactly how lactate might act as a metabolic regulator to biochemically support glucose oxidation during hypoglycemia remains unclear. Contradictory evidence suggests that increased plasma lactate levels should reduce, not augment, cerebral glucose utilization (13). The experimental lactate infusion protocol used in the study by Herzog et al. raised plasma lactate levels to 2 to $3 \mathrm{mM}$ (approximately 2- to 3 -fold above normal) in the recurrently hypoglycemic rats (15); therefore, it remains somewhat unresolved whether the results were due to recurrent hypoglycemia alone or due to $3 \mathrm{dRH}$ combined with elevated plasma lactate. Considering that lactate had little beneficial effect in control rats exposed to acute hypoglycemia, presumably it is the brain's adaptation to recurrent hypoglycemia that permits enhanced glucose metabolism during acute episodes of hypoglycemia.

The findings of augmented glucose transport/metabolism are consistent with previously hypothesized mechanisms by which recurrent hypoglycemia could contribute to hypoglycemia unawareness. Some animal models have shown an increase in brain glucose transport induced by hypoglycemia (16), but evidence in humans has been less clear. In patients with hypoglycemia unawareness or patients exposed to antecedent hypoglycemia, studies have shown that during acute hypoglycemia there is either a relatively decreased (i.e., not increased) cerebral glucose clearance and blood flow $(17,18)$ or, alternatively, that there are no changes in glucose transport, cerebral glucose metabolism, or cerebral blood flow (19). In the study by Herzog et al., the authors observed no difference in glucose transport during euglycemia between control and $3 \mathrm{dRH}$ animals, consistent with the lack of change in GLUT1 and GLUT3 glucose transporter protein expression. During hypoglycemia, however, glucose transport, which normally declines during hypoglycemia, was 
preserved in the $3 \mathrm{dRH}$ animals. The relatively high glucose transport was therefore not due to an upregulation of total glucose transporters but is speculated to be due to a change in glucose transporter function via unclear mechanisms (although possibly via increased availability of transporters at the plasma membrane). This increased capacity for glucose transport in $3 \mathrm{dRH}$ rats helped maintain intracellular glucose levels and preserve glucose metabolism during hypoglycemia. Looked at in another way, although glucose transport across the blood-brain barrier is normally rate limiting during hypoglycemia, enhanced capacity for glucose transport associated with recurrent hypoglycemia adaptations no longer limits glucose transport. Given the conflicting clinical reports, however, the possible mechanism(s) by which recurrent hypoglycemia may preserve cerebral glucose metabolism during hypoglycemia by enhancing capacity for glucose transport in the absence of changes in glucose transporter expression certainly warrants further investigation.

\section{Therapeutic implications}

Although lactate infusion has been shown to support brain function during acute hypoglycemia in people with (20) and without (21) diabetes, Herzog et al. acknowledge that lactate administration is unlikely to be used as an antecedent therapy to support brain function during moderate hypoglycemia. Of note, however, lactate treatment may be of particular use in recovering from severe hypoglycemia, for which it has been shown to prevent the vast majority of neuronal death (5).

Perhaps the more clinically relevant question is whether the recurrent hypoglycemia-induced brain adaptations that enhance lactate uptake and preserve glucose metabolism are ultimately beneficial or maladaptive. Certainly in the setting of severe hypoglycemia, brain adaptations that preserve cognition could be considered advantageous (especially when considering the alternative). Our laboratory has shown that adaptations associated with recurrent antecedent hypoglycemia protect the brain against severe hypoglycemia-induced brain damage, seizures, and cognitive decline (4). Thus, similar to the phenomena of preconditioning, recurrent bouts of moderate hypoglycemia might, paradoxically, render an individual more prone to, but less vulnerable to, an episode of severe hypoglycemia.
Unfortunately, metabolic adaptations in patients with hypoglycemia unawareness that allow cognitive function at dangerously low blood sugar levels do so at the perilous risk of a precipitous hypoglycemic coma. Since limiting the awareness and symptoms of hypoglycemia jeopardizes patient safety, it must be considered a maladaptive response. Further research into defining the mechanisms by which the brain adapts to recurrent episodes of hypoglycemia will hopefully lead to the development of therapeutic strategies that will help protect against the morbidity and mortality associated with hypoglycemia.

\section{Conclusions}

Until a cure for diabetes is found, hypoglycemia will continue to be a major barrier for the achievement of long-term glycemic control. Numerous assiduous research studies have begun to uncover the mechanisms by which the brain responds and adapts to hypoglycemia. Understanding these mechanisms will undoubtedly lead to better management and therapeutic options that will reduce the risk for hypoglycemia, while still allowing patients to achieve the benefits associated with tight glycemic control.

\section{Acknowledgments}

Research support related to this commentary was from the NIH (DK073683 and NS070235), Washington University's Diabetes Research Center (DK020579) and Nutrition Obesity Research Center (P30DK056341), and the Children's Discovery Institute of Washington University and St. Louis Children's Hospital.

Address correspondence to: Simon J. Fisher, Associate Professor of Medicine, Cell Biology and Physiology, Division of Endocrinology, Metabolism, and Lipid Research, Washington University in St. Louis, Campus Box 8127, 660 South Euclid Ave., St. Louis, Missouri 63110, USA. Phone: 314.362.3268; Fax: 314.362.7641; E-mail: sfisher@dom.wustl.edu.

1. [No authors listed]. The effect of intensive treatment of diabetes on the development and progression of long-term complications in insulin-dependent diabetes mellitus. The Diabetes Control and Complications Trial Research Group. NEngl J Med. 1993;329(14):977-996.

2. [No authors listed]. Effect of intensive blood-glucose control with metformin on complications in overweight patients with type 2 diabetes (UKPDS 34). UK Prospective Diabetes Study (UKPDS) Group.
Lancet. 1998;352(9139):1558-1558.

3. Kubiak T, Hermanns N, Schreckling HJ, Kulzer B, Haak T. Assessment of hypoglycaemia awareness using continuous glucose monitoring. Diabet Med. 2004;21(5):487-490.

4. Puente EC, et al. Recurrent moderate hypoglycemia ameliorates brain damage and cognitive dysfunction induced by severe hypoglycemia. Diabetes. 2010;59(4):1055-1062.

5. Won SJ, et al. Prevention of acute/severe hypoglycemia-induced neuron death by lactate administration. J Cereb Blood Flow Metab. 2012;32(6):1086-1096.

6. Asvold BO, Sand T, Hestad K, Bjorgaas MR. Cognitive function in type 1 diabetic adults with early exposure to severe hypoglycemia: a 16-year follow-up study. Diabetes Care. 2010;33(9):1945-1947.

7. Tanenberg RJ, Newton CA, Drake AJ. Confirmation of hypoglycemia in the "dead-in-bed" syndrome, as captured by a retrospective continuous glucose monitoring system. Endocr Pract. 2010;16(2):244-248.

8. Skrivarhaug T, Bangstad HJ, Stene LC, Sandvik L, Hanssen KF, Joner G. Long-term mortality in a nationwide cohort of childhood-onset type 1 diabetic patients in Norway. Diabetologia. 2006; 49(2):298-305

9. Cryer PE. Mechanisms of hypoglycemia-associated autonomic failure and its component syndromes in diabetes. Diabetes. 2005;54(12):3592-3601.

10. Reno CM, Litvin M, Clark AL, Fisher SJ. Defective counterregulation and hypoglycemia unawareness in diabetes: mechanisms and emerging treatments. Endocrinol Metab Clin North Am. 2013;42(1):15-38.

11. Mason GF, Petersen KF, Lebon V, Rothman DL, Shulman GI. Increased brain monocarboxylic acid transport and utilization in type 1 diabetes. Diabetes. 2006;55(4):929-934.

12. De Feyter HM, Shulman GI, Rothman DL, Petersen KF. Increased brain uptake of lactate in type 1 diabetic patients with hypoglycemia unawareness. Abstract presented at: 72nd American Diabetes Association Scientific Sessions; June 9, 2012; Philadelphia, Pennsylvania, USA.

13. Wyss MT, Jolivet R, Buck A, Magistretti PJ, Weber $B$. In vivo evidence for lactate as a neuronal energy source. J Neurosci. 2011;31(20):7477-7485.

14. Lubow JM, et al. Brain oxygen utilization is unchanged by hypoglycemia in normal humans: lactate, alanine, and leucine uptake are not sufficient to offset energy deficit. Am J Physiol Endocrinol Metab. 2006;290(1):E149-E153.

15. Herzog RI, et al. Lactate preserves neuronal metabolism and function following antecedent recurrent hypoglycemia. J Clin Invest. 2013; 123(5):1988-1998.

16. Simpson IA, et al. Blood-brain barrier glucose transporter: effects of hypo- and hyperglycemia revisited. J Neurochem. 1999;72(1):238-247.

17. Bingham EM, et al. Differential changes in brain glucose metabolism during hypoglycaemia accompany loss of hypoglycaemia awareness in men with type 1 diabetes mellitus. Diabetologia. 2005; 48(10):2080-2089.

18. Mangia S, et al. Hypoglycemia-induced increases in thalamic cerebral blood flow are blunted in subjects with type 1 diabetes and hypoglycemia unawareness. J Cereb Blood Flow Metab. 2012;32(11):2084-2090.

19. Segel SA, et al. Blood-to-brain glucose transport, cerebral glucose metabolism, and cerebral blood flow are not increased after hypoglycemia. Diabetes. 2001;50(8):1911-1917.

20. King P, Kong MF, Parkin H, MacDonald IA, Barber C, Tattersall RB. Intravenous lactate prevents cerebral dysfunction during hypoglycaemia in insulin-dependent diabetes mellitus. Clin Sci (Lond). 1998;94(2):157-163.

21. Maran A, Cranston I, Lomas J, Macdonald I, Amiel SA. Protection by lactate of cerebral function during hypoglycaemia. Lancet. 1994;343(8888):16-20. 